\title{
The Stripping Technique of Glued Cellophane Tape Mechanics Design and The Numerical Study on the Flow Field of analysis
}

\author{
Shu-Lung Wang* \\ Department of Mechanical Engineering \\ Taoyuan Innovation Institute of Technology \\ NO.414, Sec.3, Jhongshan E. Rd., Jhongli District \\ Taoyuan City, 32091 Taiwan (R.O.C.) \\ wzl@tiit.edu.tw
}

\author{
Yi-Feng Chang, Wan-te Chen \\ Department of Mechanical Engineering \\ Yuan Ze University \\ NO.135 Yuan-Tung Rd., Jhongli District Taoyuan City, \\ 32003 Taiwan (R.O.C.) \\ uno4704083@yahoo.com.tw, \\ bo882556@ms35.hinet.net
}

\author{
Jui-Yang Wang \\ Department of Information Engineering \\ National Central University \\ NO.300, Zhong Da. Rd., Jhongli District Taoyuan City, 32001 Taiwan (R.O.C.) \\ tom_yrtoo135@yahoo.com.tw
}

\begin{abstract}
In terms of environmental protection problems relating to the stripping technique of glued cellophane tape, apart from equipment demands being very high, most operators in Taiwan still manually strip the tape. The processing speed is thus slow, and cannot meet operators' expectations, which means that the industry is encountering difficulty in manufacturing and operation. In addition, due to the performance limitation of currently available tape stripping machines, the cost effectiveness is low. As for the design and analysis of the cellophane tape stripping machine, the designing of the glued cellophane tape heat transfer is an important task. The machine's heat transfer property directly affects the size of the tape stripped and the speed of the stripping. It is clearly important that a more reliable and economical tape stripping machine be developed. Moreover, in order to analyze the heat transfer problem, this study uses a novel tape stripping machine that developed and manufactured specifically for this research as the research target, and applies the simulation software FLU-ENT 5.5 to simulate the heat temperature distribution of the heat transfer on the glued cellophane tape. By using the data as a reference for the manufacture and design of the tape stripping machine, the product yield rate can be increased.
\end{abstract}

Keywords - tape stripping machine; temperature distribution

\section{INTRODUCTION}

Industrial films for the manufacturing of electronic components are indispensable for the production of multilayer ceramic capacitors used in computers and mobile phone parts. They are high-quality galvanized films, outstanding both in terms of smoothness and heat resistance. Taking the protective adhesive tapes for multilayer ceramic capacitors as an example; they are mainly used on printed circuit boards (PCB) by first sticking the protective adhesive tapes on the printed circuit board, and conducting silver immersion after the implantation of the ceramic capacitors [1]. The adhesive tapes, which are stuck to the back of the wafers, serve to protect the wafers from breaking during the cutting process. It is also necessary to secure the wafers so that they will not detach and cut the tapes while wafers plotted with circuits are cut. Recently, modular adhesive films used in the processing of multilayer chips have also been used in larger markets.

Their main function is to glue multilayer wafer slices together, and with their ingenious viscosity and cut, as well as the adhesive techniques of gluing and separation, they are indispensable in the manufacturing of tiny items. The ideal stripping conditions for the relevant adhesive tapes on wafers usually include: (1) 180-degree stripping: namely stripping the protective adhesive tapes from the surface of the wafers while keeping the stress on the wafers to the minimum. It is also applicable to the processing of thin wafers; (2) reducing damage to the circuits on the wafers: the release films used to strip the surface protective adhesive tapes are stuck to the surface by hot pressing, and the distance between them and the wafers is $3 \mathrm{~mm}$. In this way, damages to the circuits on the surface of the wafers, as imposed by sticking the release films, are reduced, and the problem of gum residuals is addressed. [3] According to discussions of process parameters, such as substrate and film thickness, the thinner the substrate and the thicker the film, the easier the stripping; while a thickersubstrate and film means a bigger area to strip. Although the use of cellophane has become more and more common, some recycling industries claim the existence of thorny "taboo"

This paper is one of the 2010 industry - university cooperative plans in terms of industrial parks organized by the Ministry of Education numbered as Q9902. Thanks to the support of the Ministry of Education, the plan could be carried out smoothly. We hereby extend our most sincere appreciation. 
substances inside the "adhesive cellophane" tapes, namely kinds of cellophane that contain the inner faces of adhesive tapes. This is why the majority of the Printed Circuit Board (PCB) industry has deemed them energy consumables, since these adhesive tapes must be torn out before they can be recycled. To make matters worse, impurities such as adhesive agents containing PMMA, release paper and release films are mixed into the raw materials of the adhesive tapes, aside from the paper, which makes tearing very difficult. Current automatic self-adhesive label stripping machines are designed to strip self-adhesive labels and their labeling, rather than being especially designed for professional protective adhesive tapes. There is also an ultra-thin ceramic paper for the "cover films" of relevant adhesive tapes used in precision instruments. The ceramic paper refers to the "dirt" that is applied on the cover films in the form of the paste, which is then heated and dried. Like baking paper used to make cakes at home, the ceramic paper can be torn from the cover film once it is dried. However, given the scale of the recycling industry for electronic resources, and the shortage of relevant research, the processing and batch production of ceramic paper cannot meet the requirements of mass production in Taiwan today. Therefore, the design focus of the equipment in this study is set to address challenges and offer breakthroughs for recycling industries.

In addition to being a response to resource and environmental demands, the cellophane tape stripping machine, as developed in this study, excels mainly in its temperature control and regulation capacities. It is able to ensure long-term continuous production, and meets the requirements of PCB manufacturers, recycling businesses, and the needs of different adhesive materials in the future, should the main trend in the PCB industry become the continuous mass production of highorder products.

\section{PROCESS AND STEPS OF DESIGN}

\section{A. General descriptions of the major structural designs and measurement systems [3]}

Selection of the heater as shown in Table I. II. Frames design, [1] as shown in Fig. 2.

TABLE I. RELATIONSHIP BETWEEN THE LENGTH OF THE HEATER AND HEATING POWER

\begin{tabular}{|c|c|c|c|c|}
\hline $\begin{array}{c}\text { Length of } \\
\text { the } \\
\text { heating } \\
\text { wire } \\
(\mathrm{mm})\end{array}$ & $\begin{array}{c}\text { Voltage } \\
(\mathrm{v})\end{array}$ & Power(w) & $\begin{array}{c}\text { Power of } \\
\text { the } \\
\text { heating } \\
\text { wire per } \\
\text { unit } \\
\text { length } \\
\text { (w/cm) }\end{array}$ & $\begin{array}{c}\text { Power } \\
\text { per unit } \\
\text { area } \\
(\mathrm{w} / \mathrm{cm})\end{array}$ \\
\hline 140 & $110-130$ & 500 & 35.7 & 40 \\
\hline 270 & $220-250$ & 1000 & 37.1 & 51 \\
\hline
\end{tabular}

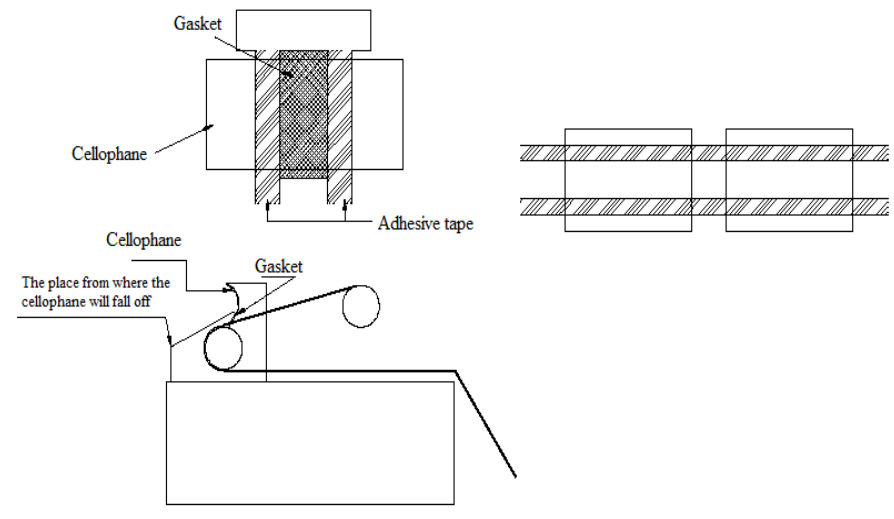

Fig. 1. Structural design plan of the adhesive cellophane tape machine

\section{RESULTS AND DisCUSSION}

\section{A. Brief descriptions of the figures}

Fig. 2 (a): the three-dimensional appearance decomposition diagram of this design

Fig. 2 (b): the three-dimensional assembled appearance diagram of this design

Fig. 2 (c): vertical three-dimensional diagram of the structural equipment units of this design

Fig. 2 (d): vertical three-dimensional diagram of the structural equipment units of this design

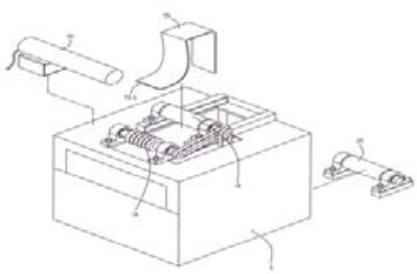

(a)

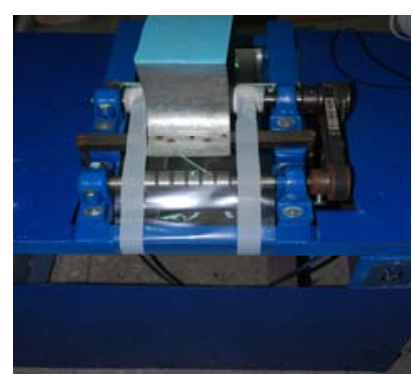

(c)

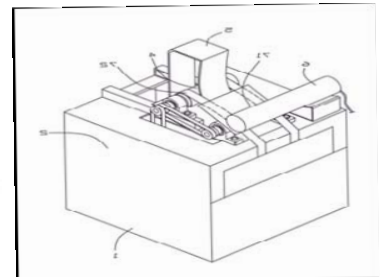

(b)

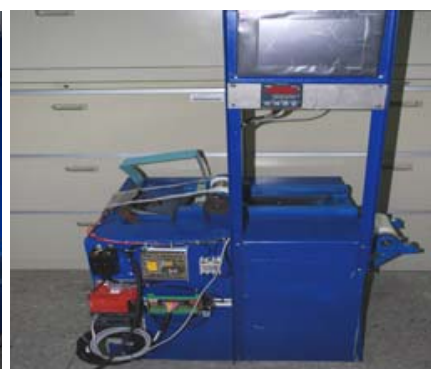

(d)
Fig. 2. (a) (b) Vertical plan of the structural equipment units of the adhesive cellophane tape machine (c) Vertical plan of the structural equipment units of the adhesive cellophane tape machine (d) Vertical plan of the structural equipment units of the adhesive cellophane tape machine

"The numbered parts in the figures"

1-machine station

2-storage roller

3-support roller 
4-retracting roller

5-baffle

51-arc-shaped port

6-air heater

71-cellophane

72-adhesive tape

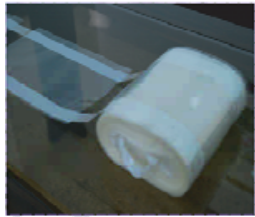

Fig. 3. Adhesive cellophane tape

The design and planning of the wiring layout and current control in the various operating units are as shown in Fig. 4.

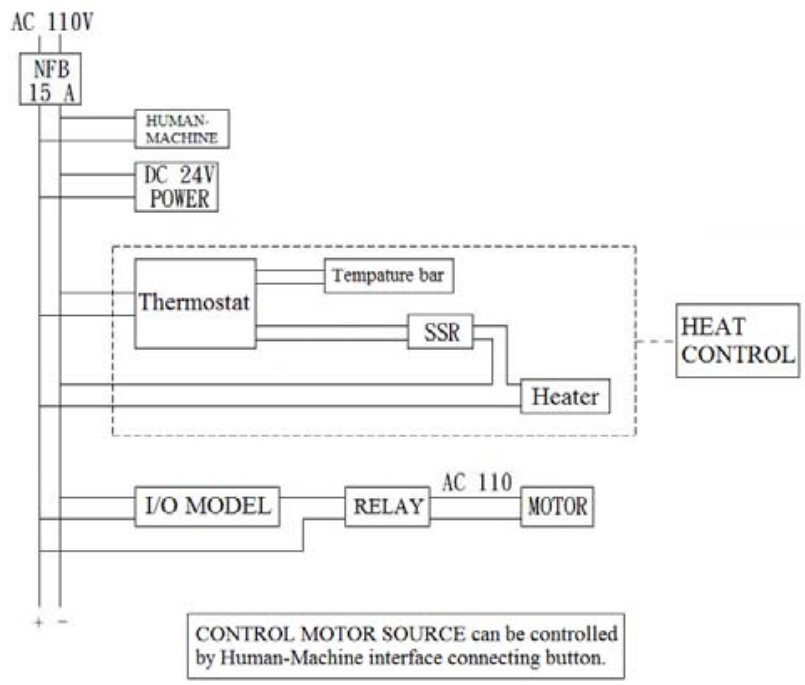

Fig. 4. Design plan of the wiring layout and current control

Description and analysis of the heat transfer on the adhesive tape $[2,3,4]$

\section{B. The temperature distribution on the surface of the adhesive tape}

Fig. 5 represents the steady-state numerical simulation of the surface temperature distribution on the adhesive tapes, and the analytical results of the optimum combination of different simulation parameters when: (the air knife has a blade angle of $45^{\circ}$, air velocity of $1 \mathrm{~m} / \mathrm{s}$, a temperature of $80^{\circ}$, and film thickness of $1.0 \mathrm{~mm}$ ).

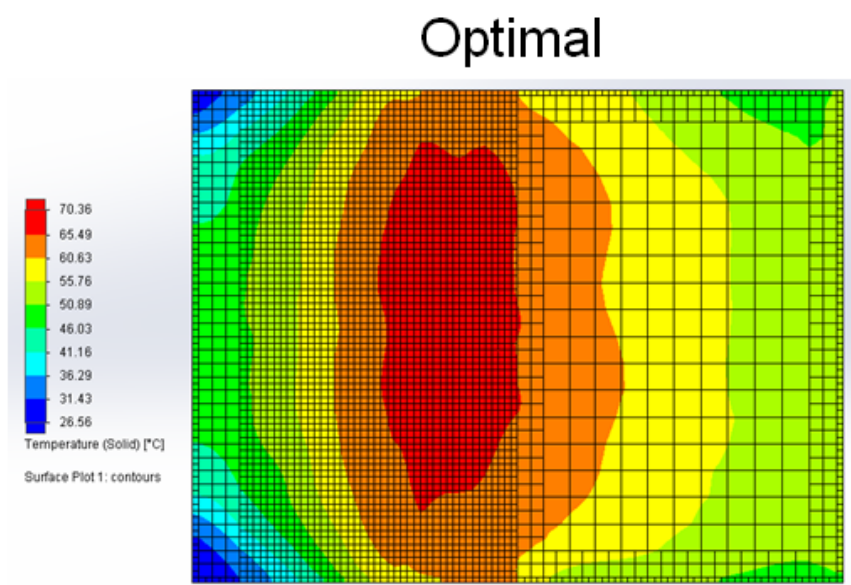

Fig. 5. Steady-state numerical simulation of the surface temperature distribution on the adhesive tapes. Analytical results of the optimum combination of different simulation parameters when: (the air knife has a blade angle of $45^{\circ}$, air velocity of $1 \mathrm{~m} / \mathrm{s}$, the temperature of $80^{\circ}$, and the film thickness of $1.0 \mathrm{~mm}$ )

\section{Numerical simulation analysis of the air velocity distribution over the flow field on the adhesive tapes}

Numerical simulation analysis of the air velocity distribution over the flow field on the adhesive tapes was conducted in this study when: (the air knife had a blade angle of $45^{\circ}$, air velocity was $1 \mathrm{~m} / \mathrm{s}$, temperature was $80^{\circ}$, and film thickness was $1.0 \mathrm{~mm}$ ). Fig. 6 shows the analysis results, as well as the surface temperature of the adhesive tapes. It can be seen that the velocity in the fields on the two sides of the air knife's air outlet are obviously lower.

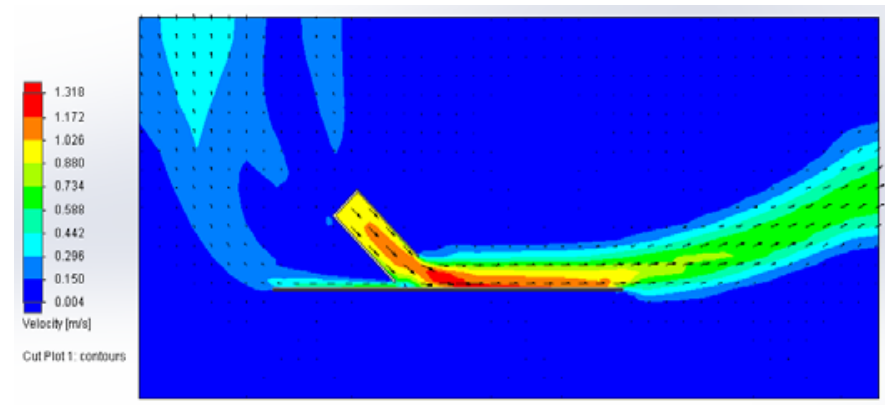

Fig. 6. Steady-state numerical simulation of the air velocity distribution over the flow field on the adhesive tapes. Analytical results of the optimum combination of different simulation parameters when: (the air knife has a blade angle of $45^{\circ}$, air velocity of $1 \mathrm{~m} / \mathrm{s}$, temperature of $80^{\circ}$, and film thickness of $1.0 \mathrm{~mm}$ )

\section{CONCLUSION}

The viscosity differences also affect the distribution density, air injection angles, air flow/air velocity, and the setting of the air injection temperatures at the outlets of the air knife. It was thus necessary to adjust the design of the air knife according to the viscosity changes in order to maintain the surface smoothness of the processed tape.

Drastic temperature increases and decreases will impose significant impacts on the quality of the cellophane and tape, 
even causing decomposition, deformation or deterioration of the materials when not properly controlled; when the temperature is too low, the separation of the tape will be impossible, leading to such deformations as ruptures and strains.

The most common reasons for ruptures and other deficiencies in the stripping process include incorrect motor speed, the viscosity of the adhesive tape, viscous drag force, tensile force of the stripping moulds, or incorrect timing or temperature settings.

This study could serve as the basis for industrial personnel to make breakthroughs in high-efficiency recycling and processing technologies domestically and abroad, establishing variable-based autonomous and standardized development technologies, and understanding the impacts on quality and functionality, as imposed by temperature control and the stripping process.

\section{REFERENCES}

[1] T.F. Wei, “Analysis of the Film Stripping Mechanism by Using Lasers”, Master's Thesis, National Cheng Kung University, June 2003.

[2] Taiwan Regional Association of Adhesive Tape Manufacturers, “Association of Adhesive Tape Manufacturers Newsletter", 20.

[3] J.G. Wang, "Design of the Adhesive Cellophane Tape Stripping Machine and Numerical Simulation Analysis of the Flow Field", Master's Thesis, Graduate School of Mechanical Engineering, Yuan Ze University, June 2014.

[4] C.K. Wang, "The Stripping Technique of Glued Cellophane Tape Mechanics Design and The Numerical Study on the Flow Field of analysis," Master's Thesis, Graduate School of Mechanical Engineering, Yuan Ze University, December 2013. 